\title{
Combination of CT and RT-PCR in the screening or diagnosis of COVID-19
}

\author{
Youxin Wang ${ }^{1,2}$, Haifeng Hou ${ }^{3}$, Wenrui Wang ${ }^{2}$, Wei Wang ${ }^{1,4}$
}

\footnotetext{
${ }^{1}$ Beijing Key Laboratory of Clinical Epidemiology, School of Public Health, Capital Medical University, Beijing, China

${ }^{2}$ Inner Mongolia Comprehensive Center for Disease Control and Prevention, Hohhot, Inner Mongolia Autonomous Region, China

${ }^{3}$ School of Public Health, Shandong First Medical University \& Shandong Academy of Medical Sciences, Taian, Shandong Province, China

${ }^{4}$ School of Medical and Health Sciences, Edith Cowan University, Perth, Australia
}

Neither chest CT nor RT-PCR testing alone is accurate enough for the diagnosis of COVID-19 infection.

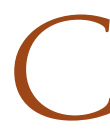
oronavirus Disease 2019 (COVID-19), emerged in Wuhan, China, in December 2019. As of 10 March 2020, COVID-19 cases have been reported in 114 countries from all Continents except Antarctica, with accumulative 80932 cases in China and 29432 in other countries [1]. The transmission potential of

COVID-19, determined by reproduction number (R0) of 3.28, is much higher that of severe acute respiratory syndrome (SARS) [2]. Bold measures taken by China have effectively curbed the rapid spread of this new respiratory disease source and changed the dangerous process of rapid spread of the epidemic [3]. The world is not yet ready to organize and implement the measures that have been proved to be efficient and effective by China to block or minimize the spread of new coronavirus [3]. The crude case-fatality rate (CFR) of COVID-19 is reported to be $2.3 \%$ in all patients [4], while higher to be $61.5 \%$ in critically ill patients [5]. Therefore, early screening and quarantining mild or asymptomatic cases and early diagnosis of sever patients for intensive treatment are urgent to avoid the pandemic of COVID-19.

Reverse Transcription-Polymerase Chain Reaction (RT-PCR) testing was recommended to confirm COVID-19 cases by China medical authority [6], however the total positive rate of RT-PCR for throat swab samples was about 30\% to 60\% at initial presentation. Chest Computed Tomography (CT), a routine imaging method, has also been applied to diagnose COVID-19 infection [7]. A study on the correlation of chest CT and RT-PCR testing of COVID-19 based on 1014 cases demonstrated that the sensitivity of chest CT imaging for COVID-19 was $97 \%$ (580/601), and the specificity was $25 \%$ (105/413), with RT-PCR as a diagnosis criterion [8]. As a new emerging infectious disease, there is no gold criteria for the diagnosis of COVID-19. Our current study showed that if chest CT was taken as a reference of diagnosis standard, the sensitivity of RT-PCR

Parallel tests with CT and RT-PCT are recommended to be applied in the screening of COVID-19 for isolation, while series test with CT or RT-PCT should be used in COVID-19 diagnosis confirmation for treatment. 
Table 1. The performance of RT-PCR for COVID-19 infection with chest CT result as reference

\begin{tabular}{|c|c|c|c|c|}
\hline \multirow[t]{2}{*}{ CT } & \multicolumn{2}{|c|}{ RT-PCR } & \multirow{2}{*}{$\begin{array}{l}\text { Sensitivity } \\
\text { (95\% CI) }\end{array}$} & \multirow{2}{*}{$\begin{array}{l}\text { SPECIFICITY } \\
\text { (95\% CI) }\end{array}$} \\
\hline & positive & NEGATIVE & & \\
\hline Positive & 580 & 308 & \multirow{2}{*}{$\begin{array}{c}65 \% \\
(62 \%-68 \%)\end{array}$} & \multirow{2}{*}{$\begin{array}{c}83 \% \\
(76 \%-89 \%)\end{array}$} \\
\hline Negative & 21 & 105 & & \\
\hline
\end{tabular}

CT - computed tomography, RT-PCR - reverse transcriptase polymerase chain reaction, $\mathrm{CI}$ - confidence interval testing for COVID-19 was 65\% (580/888), and the specificity is $83 \%$ (105/126) (Table 1). Thus, if both sensitivity and specificity were taken into account simultaneously, neither chest CT nor RT-PCR testing alone is accurate enough for the diagnosis of COVID-19 infection.

Considering that asymptomatic cases are also of higher transmission potential, sensitivity should be first considered for the screening purpose of COVID-19 infection. When confirmation for intensive treatment, specificity should be first consid-

ered to avoid false treatment. Parallel tests and serial test are needed to increase both sensitivity and specificity. Parallel tests perform RT-PCR and CT imaging at the same time and the results are cross-referenced to make the diagnosis [9]. Serial test employs as a secondary screening test which is performed only if the result of initial screening test is positive [9]. For screening purposes, parallel tests, ie, positive in either RT-PCR or chest CT is used to clinically diagnose COVID-19, can improve sensitivity and decrease false negative cases. For therapy purposes serial tests should be used to improve specificity and decrease false positive cases. Consequently, we recommend that parallel tests are used in screening, while series tests should be used for diagnosis confirmation of COVID-19. The proposed strategic approach for screening and diagnosis confirmation of COVID-19 infection might also be of reference significance for other countries or other emerging infectious disease.
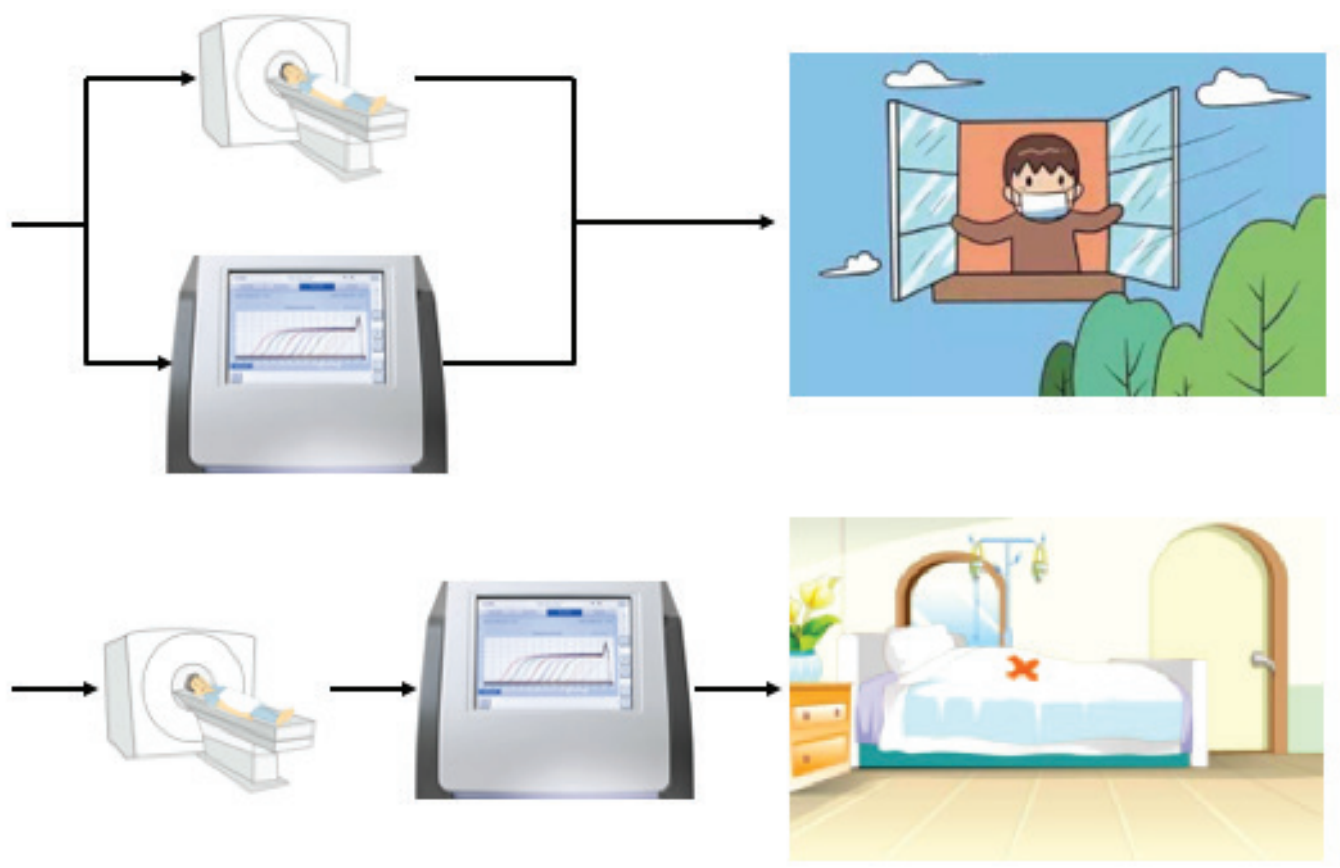

Photo: Parallel or serial test (from the authors' own collection, used with permission).

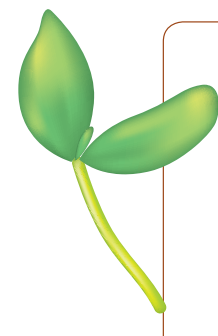

Funding: The study is supported by the National Natural Science Foundation of China $(81673247,81703317$ and 81872682).

Authorship contributions: Youxin Wang \& Wei Wang: design, draft, and revise the manuscript. Haifeng Hou \& Wenrui Wang: data analysis.

Conflict of interest: The authors completed the ICMJE Unified Competing Interest form (available upon request from the corresponding author), and declare no conflicts of interest. 
1 National epidemic situation. [In Chinese].Available: https://news.qq.com/zt2020/page/feiyan.htm. Accessed: 10 March 2020.

2 Liu Y, Gayle AA, Wilder-Smith A, Rocklov J. The reproductive number of COVID-19 is higher compared to SARS coronavirus. J Travel Med. 2020;27:taaa021. Medline:32052846 doi:10.1093/jtm/taaa021

3 China-WHO Joint Expert Team. Joint investigation report on Novel coronavirus pneumonia (COVID-19). Available: http:// www.nhc.gov.cn/jkj/s3578/202002/87fd92510d094e4b9bad597608f5cc2c/files/e73a238d8eff45d5ab855fa078f4c0dd. pdf. Accessed: 10 March 2020.

4 Ebrahim SH, Ahmed QA, Gozzer E, Schlangenhauf P, Memish YA. Covid-19 and community mitigation strategies in a pandemic. BMJ. 2020;368:m1066. Medline:32184233 doi:10.1136/bmj.m1066

5 Yang X, Yu Y, Xu J, Shu H, Xia J, Liu H, et al. Clinical course and outcomes of critically ill patients with SARS-CoV-2 pneumonia in Wuhan, China: a single-centered, retrospective, observational study. Lancet Respir Med. 2020; pii: S22132600(20)30079-5. [Epub ahead of print]. Medline:32105632 doi:10.1016/S2213-2600(20)30079-5.

6 General Office of National Health Committee. Notice on the issuance of a program for the diagnosis and treatment of novel coronavirus (2019-nCoV) infected pneumonia (trial sixth edition) (2020-02-18). Available: http://www.nhc.gov.cn/ yzygj/s7653p/202002/8334a8326dd94d329df351d7da8aefc2/files/b218cfeb1bc54639af227f922bf6b817.pdf. Accessed: 24 February 2020.

7 Yang Y, Yang M, Shen C, Wang F, Yuan J, Li J, et al. Evaluating the accuracy of different respiratory specimens in the laboratory diagnosis and monitoring the viral shedding of 2019-nCoV infections. MedRxiv. 2020. doi:10.1101/2020.02.11.2 0021493.

8 Ai T, Yang Z, Hou H, Zhan C, Chen C, Lv W, et al. Correlation of chest CT and RT-PCR testing in coronavirus disease 2019 (COVID-19) in China: A report of 1014 cases. Radiology. 2020. 200642. Epub ahead of print. Medline:32101510 doi:10.1148/radiol.2020200642

9 Lei X, Wang J, Yang Z, Zhou S, Xu Z. Diagnostic value of pleural effusion mononuclear cells count and adenosine deaminase for tuberculous pleurisy patients in China: A case-control study. Front Med (Lausanne). 2019;6:301. Medline:31921874 doi:10.3389/fmed.2019.00301

\section{Correspondence to:}

Wei Wang, MD, PhD, FFPH, FRSB, FRSM

School of Medical and Health Sciences

Edith Cowan University

270 Joondalup Drive

Perth 60127

Australia

wei.wang@ecu.edu.au 